\title{
Indoor Atlas Service as a Tool for Building an Interior Navigation System
}

\author{
Martin Štancel, Ján Hurtuk, Michal Hulič, Jakub Červeňák
}

Department of Computers and Informatics, Faculty of Electrical Engineering and Informatics, Technical University of Košice

Letná 9, 04001 Košice, Slovak Republic

martin.stancel@tuke.sk, jan.hurtuk@tuke.sk, michal.hulic@tuke.sk, jakub.cervenak@student.tuke.sk

\begin{abstract}
Indoor navigation is a technology challenge relatively difficult to tackle. This paper examines and describes the implementation of a new approach to indoor navigation using the IndoorAtlas service, which was used to design and implement a prototype application. This is based on the positioning of mobile phone devices, and with the help of the aforementioned service, specific properties of the building are obtainedthese include the magnetic fingerprints, a result of the interaction of the Earth's magnetic field with the ferromagnetic materials used in the construction of buildings. In our case, this service was used in correlation with the Java programming language for the Android platform. The publication also contains experiments carried out on the premises of the selected building. Several main measures were made. In the selected environment, the completed measurements demonstrated, that the proposed application fullfils the criteria of a fully functional indoor navigation, with average deviation within the parameters specified by the manufacturer.
\end{abstract}

Keywords: indoor navigation; IndoorAtlas; geomagnetic localization; shortest path algorithms

\section{Introduction}

Over the past few decades, satellite positioning systems have proven to be a reliable and highly accurate outdoor navigation tool. This trend has forced the market to focus also on indoor positioning, to allow navigation in buildings and similar spaces. Even today, this kind of navigation is still a big challenge and there are still no reliable and sufficiently precise technical solutions in this respect. Therefore, the question is: what makes outdoor and indoor navigation so different, when - in theory - most positioning systems use a similar principle and could be used not only for outdoor but also for indoor purposes. In fact, the indoor use of outdoor positioning systems presents completely different and inconsistent results, 
and, in certain cases, outdoor navigation systems cannot even be applied indoors. From a technical point of view, indoor navigation is much more complex due to the reasons as objects in the rooms, such as furniture and the walls themselves, contribute to significant signal reflections, non-life-of-sight conditions [1], high attenuation, dispersion and possible reflection of the signal due to higher obstacle density, short-term signal changes due to the presence of humans, pets, etc. (repositioning of furniture, new furnishings, opening and closing of doors, etc.) and very high demands on measurement accuracy.

On the other hand, it can be stated that the issue of indoor positioning also has some positive aspects. The area used for positioning is significantly smaller. It can be stated, that temperature fluctuations are not as significant, for normally, room temperature is within a certain - almost constant - range. The geometry of the mapped rooms - with the exception of furnishings and other inserted objects can be considered as simple (perpendicular walls, etc.). Compared to car-based navigation systems, it uses slower speeds and dynamics - by at least an order of magnitude.

From a research point of view, indoor navigation is also of great interest due to the lack of sufficient indoor results produced by today's dominant outdoor positioning technology - GNSS. There is no indoor positioning system achieving the excellent global outdoor results of GNSS, high accuracy, short signal delays, high availability, high-level of integration and low setup costs. Several different approaches have been made including Chaur-Heh Hsieh At al. [2] who developed four deep neural networks implemented with multi-layer perceptron (MLP) [3] [4] and one-dimensional convolutional neural network (1D-CNN) to estimate the location of a subject in a room.

Creating a navigation system to overcome the aforementioned deficiencies requires a device - in this case, an intelligent device (mobile phone, tablet) containing the necessary sensors to determine the location while guaranteeing users the simplicity, comfort, availability and - especially - the accuracy of measurement. Most modern phones have a built-in accelerometer, light sensors, and sensors capable of detecting magnetic fields [5]. These sensors are monolithic and mutually independent, unlike sensors connected into a network of distributed, mutually dependent or mutually interacting sensors. These built-in sensors have great potential for developing diverse applications.

The purpose of this paper is to present the proposed indoor navigation system for the Android platform, having low setup and operating costs. The presented application would allow to determine the indoor position of the user and navigate users through the indoor space, according to the selected parameters. The proposed indoor navigation solution will consist of two logically separated parts: device positioning and calculation of the shortest route from source to destination. Development of the aforementioned interior navigation system can be divided into several steps: 
- analysis of available technology used for indoor positioning,

- $\quad$ analysis of suitable shortest-path-calculation algorithms,

- design of an indoor navigation solution using the IndoorAtlas API [6], based on magnetic fingerprinting - mapping of unique magnetic profiles resulting from the interaction of the Earth's magnetic field with steel and other ferromagnetic materials used in the construction of modern buildings,

- implementation of the proposed solution,

- evaluation of functionality using real-world testing.

\section{Related Works}

The technology used so far to solve the problem of indoor positioning can be divided into three categories, representing three different physical principles as inertial positioning using an accelerometer and a gyroscope to meter angular momentum, positioning technology based on mechanical waves using (ultra)sound or positioning technologies based on electromagnetic waves using optical, infrared, microwave and radio spectrum. Several papers have been developed to research these various approaches, document their pros and cons, and ultimately develop systems based on previously mentioned technologies in order to confirm the main functionality. Detailed comparisons of the existing technologies are made in [11], [19], [21], [22].

\subsection{Positioning Technologies Using Camera Systems}

Optical indoor positioning systems can be categorized as egomotion systems [7] obtaining the position of a moving camera, or static systems in which the camera determines the positions of objects moving in the images. As the application is designed for smartphones supporting the Android platform, we will focus on egomotion systems. One of these systems is the Mobile Visual Indoor Positioning System [8] using a distributed architecture. The mobile application creates photos of its surroundings and then sends them to a server that compares the submitted photos with a database of properly localized and oriented photographs of the environment, taken during the initial calibration phase. The server component uses the Speeded Up Robust Features comparison algorithm [9], which provides fast, memory-efficient extraction and description of recognition points from a local photograph. After executing the algorithm, the server component has the most probable photograph, including points of interest and a reference photograph stred in the database. Since the position of the reference photo, stored in the database, 
usually differs from the position where the current photograph was taken from, a geometric correction scheme is applied. This is based on the application of a formula stating that the distance between two photographs of the same object taken from different distances from the object is proportional to the distance of the two points on the photograph. The mobile app does not depend on specific infrastructure. The whole system can be presented as cheap and relatively efficient. On the other side, the system for its functionality needs a series of photos with a high amount of recognizable points for exact positioning. Therefore, it is not sufficient to use it with positioning situated in open empty spaces. Another disadvantage lies in bad light conditions presented on mentioned photographs and in situations, where the scene can be often changed (e.g. furniture position changing).

\subsection{Positioning Based on LED Lighting}

A solution based on this technology is Philips Indoor Positioning. This system uses LED lamps producing energy-efficient and high-quality light, while using Philips Visible Light Communication [10] technology to transmit unique codes [11], which are then detected by the smartphone. The accuracy of positioning depends on the accuracy of the light sensor tilt angle metering. This system modulates the intensity of the optical LED light to transmit data and receives light through the light sensor of the mobile phone. Data from different light sources can be received at the same time. This is possible due to the spatial separation of the light rays emitted by various LED sources using lenses, provided that the light sensor is directed towards the light source. The system also consists of the control software and the cloud solution, which can identify code and therefore computes the exact position of the smartphone. Aside from the system mentioned above, Philips comes with Indoor Positioning System, which uses BTLE (Bluetooth Low Energy) sensors. These sensors are used to in-pocket notifications for route tracking, even without the need for active use of the mobile phone [11]. Positioning based on LED lightning represents one of the most reliable solutions because the radio and electromagnetic interferences do not have any impact on system stability. One of the huge disadvantages lies, however, in relatively huge costs connected to realization.

\subsection{Positioning Mechanism Using Inertial Force}

This system [12] is based on the capabilities of modern mobile phones equipped with an accelerometer, a compass, a camera, and an Internet connection. The data read from the smartphone sensors, in conjunction with the location map, provides the current position independently of the pre-installed positioning systems using the Dead reckoning technique [13]. 
After starting the application, the user uses the integrated camera to scan the QR code located on the particular floor. Based on the URL encoded in the QR code, the application will download an indoor vector map of the particular floor from the dedicated server along with the user's initial position on the map, corresponding to the position where the user captured the $\mathrm{QR}$ code. If the user moves, the application will draw its location as a continuous line on the downloaded floor plan, step-by-step. Using the data generated by the smartphone accelerometer, the system records the number of steps taken by the user. A step is detected as a pair of positive and negative values returned by the accelerometer. The current user orientation is determined by the digital compass. The initial orientation is determined when scanning the code, perpendicular to the floor plan. The whole system can be considered as less effective due to several factors. Length of the step is computed based on step acceleration force. Therefore, the end-user can with simulated movements force the system to count imaginery steps and eventually disrupt the computation. Also, occurring errors are cumulative and are increased with every next step of the end user [12].

\subsection{Positioning Using Ultrasound or Radio Waves}

This solution is based on the use of sound and radio waves to accurately determine the location of the user. As one of the practical examples can be stated is the system based on Wi-Fi signal. For the functionality of the system [14], beacons static units installed on the ceilings - are required. The ultrasonic beacon periodically emits ultrasonic pulses and radio frequency messages with a unique identification code. By applying the Time-of-Arrival principle [15] on receiving information from another beacon and the speed of sound corrected for indoor air temperature, the receiver calculates its distance from the respective beacons. Since radio frequency messages propagate through space $10^{6}$ times faster than ultrasound, the receiver calculates the distance of the receiver to the beacon based on the time elapsed between the start of the radio frequency message and the corresponding ultrasonic impulse. The receiver position can then be determined based on the coordinates of the beacons and the measured distances. Then, using trilateration or multilateration, it is possible to calculate the position of the smartphone in space, knowing the distances to several known beacons. However, using this system to track the end user can be considered as not suitable because the ultrasound is sensitive to temperature fluctuations and multipath signals bouncing.

\subsection{Positioning Techniques Utilizing Magnetic Fields}

Modern buildings containing steel and other ferromagnetic materials in their structures have unique, spatially dependent magnetic fingerprints that can be used for positioning purposes. Unique magnetic fields arise from the interaction of the 
Earth's magnetic field with ferromagnetic materials. The non-constant surrounding magnetic fields produce different magnetic profiles, depending on the path taken by the user. One of the systems using this principle for positioning is IndoorAtlas. The application uses the IndoorAtlas API to communicate with the positioning service. The API sends the processed data of the smartphone sensors to the positioning service, which then calculates the current position and forwards it back to the applications via the API. This technology represents an innovative indoor positioning approach that can be used on its own or in collaboration with other technologies to improve accuracy. IndoorAtlas is a software positioning service that - in terms of hardware - requires only a smartphone equipped with built-in sensors to operate. In modern buildings, the accuracy of this technology ranges from $0.1 \mathrm{~m}$ to $2 \mathrm{~m}$ [6]. The service itself is based on the Microsoft Windows Azure Cloud platform. Before using the service, it is needed to generate a magnetic map of the indoor spaces, which are intended to be navigated through. Indoor Atlas has come with a complete solution including:

- IndoorAtlas Plans - web application designed to use for adding and managing individual floor plans,

- IndoorAtlas Map Creator - mobile app designed to measure magnetic fields and uploading measured values into the database,

- IndoorAtlas API - serves as a mediator for extracting the location.

\section{Algorithms for Finding the Optimal Path}

In navigation systems, it is necessary to determine the most optimal path from node $\mathrm{A}$ to node $\mathrm{B}$ passing through nodes $\mathrm{X}_{0} \ldots \mathrm{X}_{\mathrm{n}}$. Graph algorithms whose core is common are used for this. As the navigation system is to serve only in indoor conditions, algorithms that look for the shortest path in an unoriented edge-rated graph. Routing algorithms scan graphs from the starting node through neighboring nodes until they reach the destination [20].

\subsection{Dijkstra's Algorithm}

Dijkstra's algorithm stores all nodes in the priority queue sorted by distance from the source - in the first iteration, only the source has a distance of 0 , all other nodes are infinite. In each step, the algorithm selects the highest priority node from the queue, which is the node with the lowest distance from the already processed part and places it among the processed nodes. It then goes through all of its unprocessed descendants, adds them to the queue if they are not already occupied, and verifies that they are no closer to the resources than they were before they were processed. The algorithm terminates when all nodes are 
processed. The total asymptotic time complexity of Dijkstra's algorithm is $\mathrm{O}\left(\mathrm{n}^{2}\right)$ [20].

\subsection{Floyd-Warshall Algorithm}

The Floyd-Warshall algorithm has a distance matrix at the input, let's call it $\mathrm{D}^{0}$. If the edge of length 1 leads between two nodes $(i, j)$, then this matrix contains exactly this value on the index $(i, j)$. On the diagonal, this matrix has only zeros, and on other indices that do not correspond to an infinity edge. In other words, this matrix contains the distances of the nodes that do not pass through any intermediary. In each iteration of the Floyd-Washall algorithm, this matrix is recalculated to express the distance of all pairs of nodes through a gradually increasing set of potential intermediaries. Simply put, matrix $\mathrm{D}^{1}$ will express the distance of all nodes with the possibility of using one (given) intermediary, $\mathrm{D}^{2}$ distance with possible use of two (given) intermediate nodes, $\mathrm{D}_{\mathrm{m}}$ with the possibility of using $m$ intermediate nodes. Because the values from which the elements of the new matrix are calculated cannot be overwritten during the transformation, it is enough to use exactly one matrix for $D^{i}$ and $D^{i+1}$ in the implementation itself. The total asymptotic time complexity of Dijkstra's algorithm is $\mathrm{O}\left(\mathrm{n}^{2}\right)$ [20].

\subsection{Bellman-Ford Algorithm}

The Bellman-Ford algorithm uses the edge relaxation method, which determines the currently set value of the shortest distance from node $\mathrm{S}$. If the value at node is found to be higher than the value from the current node plus the edge rating from the current node to the node we would like to change its value, then the change (decrease) of this value is made. The main difference from Dijkstra's algorithm lies in the way passage chart. If all successors of one node using Dijkstra's algorithm are passed, then this node is closed and then the algorithm no longer modifies it. This does not happen in the Bellman-Ford algorithm, as it does not close these nodes immediately, but passes all nodes several times and gradually adjusts the values of the shortest path distances. The complexity of the algorithm is $\mathrm{O}(V . E)$, where $\mathrm{V}$ represents a number of nodes and $\mathrm{E}$ number represents of edges [20].

\subsection{Conclusion}

The dynamics of interior conditions can be considered relatively low. The Bellman-Ford algorithm makes it possible to find the shortest path in a graph that also contains negative edges, which is irrelevant for our system, as the distances between individual nodes should correspond to the real distance between the given geographical coordinates. 
The Floyd-Warshall algorithm provides finding the shortest path from any starting node to any end node in a single run, which is redundant for our system and would be wasted on performance. Finding the shortest path from one given start node to a single end node is suitable for proposed system and therefore the use of Dijkstra's algorithm is the optimal solution for the proposed system.

\section{Comparison of Indoor Position Technologies}

Mobile phone camera localization is not the optimal way to obtain a location for a presented solution. The use of cameras would not guarantee the reliable operation of the proposed system in poor lighting conditions, or in buildings with large rooms, where the system would have difficulty identifying a sufficient number of recognition points.

To determine the position using Philips Visual Light Communication technology, it is necessary to install LED lights in every place in the interior where we want to obtain the position of the mobile phone. The use of this technology would ensure accuracy and reliability, but for the presented system, we need to find a solution that has the lowest possible implementation costs and does not require any additional installation of equipment. Therefore, the use of this technology is not again suitable for the presented system.

Another option lies in localization, which uses the accelerometer and compass of the smartphone to calculate the position of the device. It is not necessary to have additional hardware for the proper functioning of this technology, which can be considered asi a big advantege. However, calculating the distance from the initial position using an accelerometer to estimate the step length is a source of large error in determining the exact position. The user could also simulate walking with certain movements, which would lead to a malfunction of the system. This technology is inaccurate and therefore, it cannot be considered suitable for integration into the proposed system.

There are several limitations to calculate the positions using ultrasound and radio waves (e.g.). The system is sensitive to temperature fluctuations and multiway signal reflection. The operation of the system requires the installation of beacons that transmit ultrasound and radiofrequency messages. All these negatives make this technology unreliable and unusable for our system.

When using a system that would determine the user's position using bluetooth beacons, the installation of bluetooth beacons would be required for the system to work. To achieve good results, it would be necessary to install these lighthouses in the vicinity distances and so as to cover the whole area in which we want to locate the user. This technology is expensive and again unnecessarily complicated for the proposed system. 
Use the unique magnetic field in the interior to determine the position of the mobile device is a technology whose installation costs are virtually zero. This technology is represented by IndoorAtlas. No installation of equipment is required to implement this solution. This technology is able to work in a wide variety of conditions. Only a smartphone equipped with sensors that are able to record the values of the magnetic field in individual parts of the mapped space is needed for operation. The used smartphone needs only Internet access to receive updates about the current location. The positioning accuracy of this system varies in the range of 0.1 meters to 2 meters. Due to the low implementation costs, sufficient accuracy, and efficiency, this technology is the optimal solution for a given problem.

Table 1 presented below compares the main features of previously stated technologies, which can be considered as essential for the further proposed system.

Table 1

Comparison of the selected technologies [11] [19] [21] [22]

\begin{tabular}{|l|c|c|c|c|c|}
\hline & $\begin{array}{c}\text { Bluetooth } \\
\text { Beacons } \\
\text { Positioning } \\
\text { Technology }\end{array}$ & $\begin{array}{c}\text { Wi-Fi or } \\
\text { Ultrasound } \\
\text { Based } \\
\text { Solution }\end{array}$ & $\begin{array}{c}\text { IndoorAtlas } \\
\text { Solution }\end{array}$ & $\begin{array}{c}\text { Philips Visual } \\
\text { Light } \\
\text { Communication } \\
\text { Solution }\end{array}$ & $\begin{array}{c}\text { Egomotion } \\
\text { Systems }\end{array}$ \\
\hline $\begin{array}{l}\text { Location } \\
\text { precision }\end{array}$ & $2 \mathrm{~m}$ & $5 \mathrm{~m}$ & $0.1 \mathrm{~m}-2 \mathrm{~m}$ & $5 \mathrm{~cm}$ & $5 \mathrm{Cm}-$ \\
\hline Maintenance & Average & Average & Easy & Easy & Average \\
\hline $\begin{array}{l}\text { Installation } \\
\text { costs }\end{array}$ & $\begin{array}{c}\text { Average / } \\
\text { High }\end{array}$ & High & Low & Average / High & Varies \\
\hline $\begin{array}{l}\text { Signal } \\
\text { change } \\
\text { sensitivity }\end{array}$ & Sensitive & Sensitive & $\begin{array}{c}\text { Sensitivity } \\
\text { based mainly } \\
\text { on electronic } \\
\text { noise / fast } \\
\text { moving metal } \\
\text { objects } \\
\text { (elevators) }\end{array}$ & Sensitive & $\begin{array}{c}\text { Sensitive to } \\
\text { light } \\
\text { conditions } \\
\text { and } \\
\text { frequently } \\
\text { changing } \\
\text { environment }\end{array}$ \\
\hline
\end{tabular}

\section{Proposed System Architecture}

The most important element of the presented system is to divide the project itself into logical partitions, separating the retrieval of the indoor position using data from the IndoorAtlas API [6] and obtaining the smallest route using Dijkstra's algorithm [7]. The following figure shows the distribution of these logical parts of the project. 


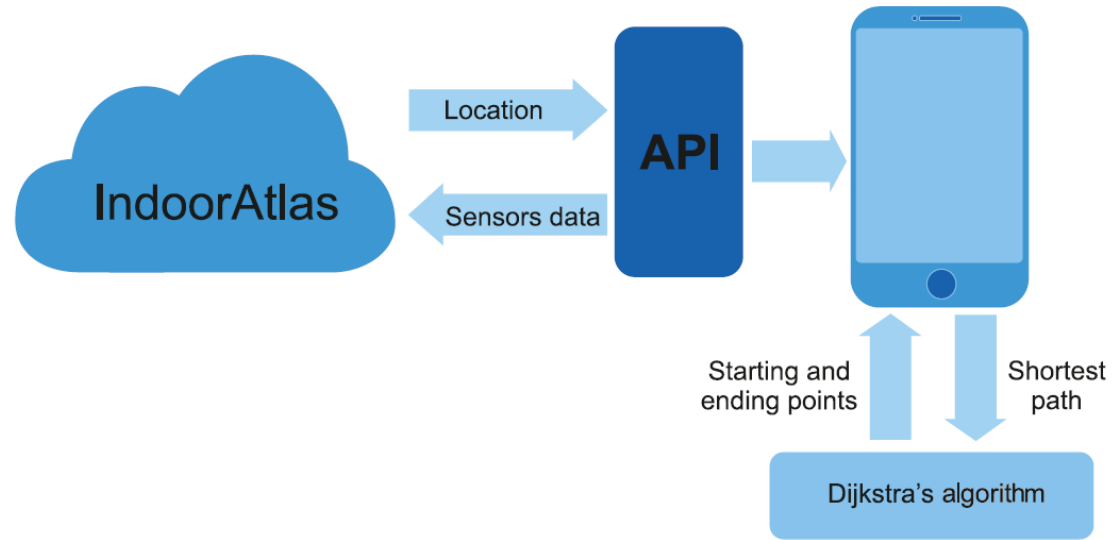

Figure 1

The proposed system architecture

The application operation principle is shown in Figure 2; this also depicts various situations that may arise when the user interacts with the application:

- a point inside the building is set as the starting point of the user's route,

- the current position is set as the starting position upon initiating navigation.

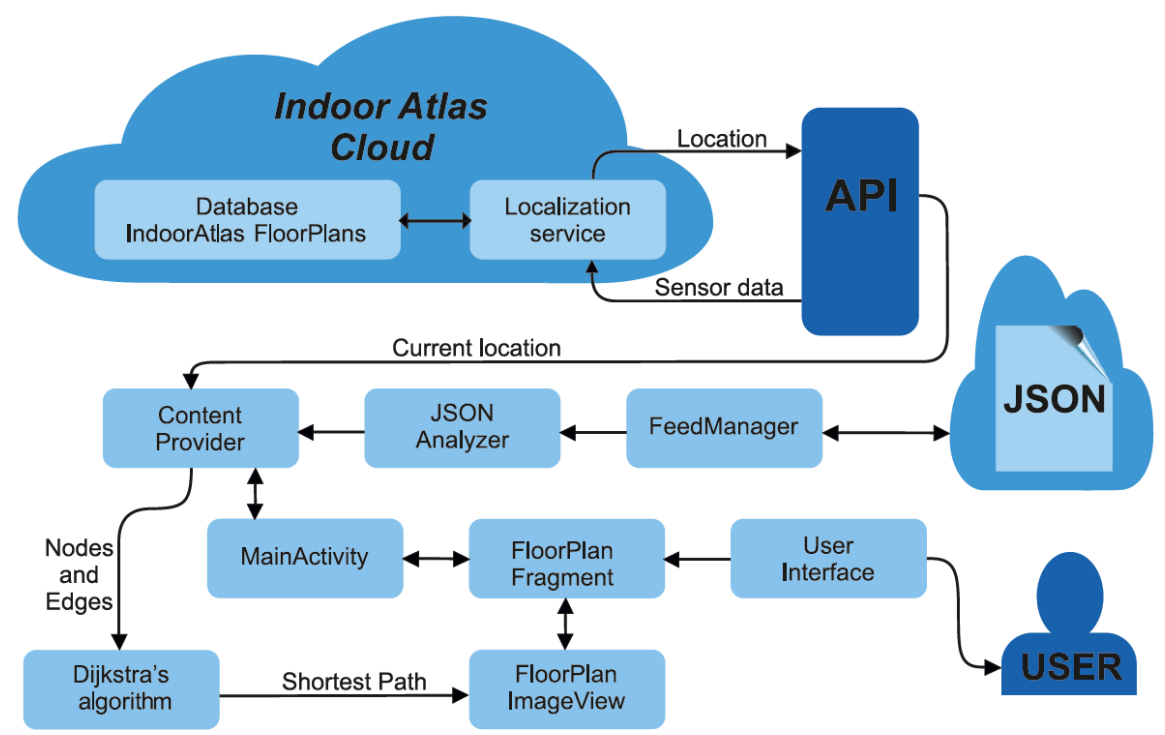

Figure 2

Basic principles of the proposed application 
- In the first case, Dijkstra's algorithm determines the shortest distance between two points, while determining the user's position is not necessary because the algorithm uses only the vertices and edges of the processed graph as its input. The graph data can be accessed via an HTTP request, which returns a JSON object that is then analyzed by the analyzer, storing all necessary object references in memory. This method allows fast run-time access to files and data.

- In the second case, the IndoorAtlas API determines the current position as the starting position when the application starts. Again, Dijkstra's algorithm is being used, so a vertex with the latitude and longitude information of the user has to be added to the graph. In this case, we also need to know the floor where the user of our application is located.

\section{Positioning with IndoorAtlas}

The IndoorAtlas API provides positioning options using magnetic sensors of smart devices in several ways:

- a map of the particular building floor is added to the GPS coordinates,

- when collecting data during the calibration phase,

- when calculating the position itself.

[6] states that the source of the earth's magnetic field is highly conductive liquid iron rotating around the outer core of the Earth. The magnetic field generated by these magnetic currents is magnetic field $B$. The magnitude of magnetic field $B$ is based on the surface area and can be described as a variable magnetic density.

The relationship between these is as follows:

$1 \frac{W b}{m^{2}}=1 T=10000 G a$

The magnetic sensor of the smart device measures the variable magnetic density, and, based on this information, determines the direction of the cardinal direction: north. It is very important to note that the north of the magnetic field does not equal the geodetic north of Earth. Geodetic north points to the north pole that Earth rotates around, while magnetic north is the position of the geomagnetic field. This position is beyond the axis of the globe. The angle between the geodetic north and the magnetic north is the magnetic declination [16].

The magnetic declination keeps changing and varies depending on the position on the globe. The total intensity of the magnetic field F consists of the X (North), Y 
(East), and Z (Down) components. The horizontal part $\mathrm{H}$ consists only of the components $\mathrm{X}$ and Y. Slope I describes the angle between H and F. Slope D is equal to the angle as shown in Figure 3.

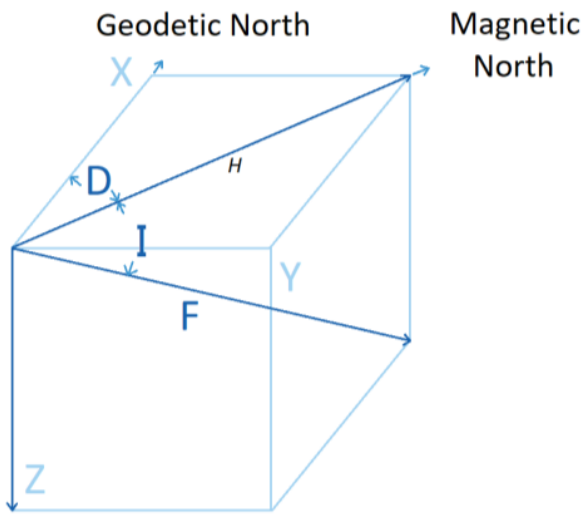

Figure 3

Parameters of the Earth's magnetic field

\section{Optimal Path Calculation}

To calculate the shortest distance between two points in real-time, Dijkstra's algorithm was used; the first point is the starting point of the positioning and the second point is the position the user wants to navigate to.

This algorithm is one of the basic algorithms in graph theory [7]. In the proposed system, the real world is mapped to a graph of vertices and edges on which Dijkstra's algorithm performs calculations. The application then communicates with the server using a JSON object, the date of which is then used to analyze the information stored in the content provider. The graph extracts the vertices and edges of the real world from the database.

\subsection{Assignment of the Building Floor Plan to GPS Coordinates}

The user position acquisition process begins by adding the building floor plan - in PNG or JPEG format - to IndoorAtlas Maps using the IndoorAtlas FloorPlans web application. This application allows easy assignment of building floor plans to the corresponding geographic coordinates, which allows the use of the geographic coordinate system in the application. To increase positioning accuracy, it is very important to set the length and width of the building to its actual dimensions, as IndoorAtlas actually calculates the distances relative to these 
dimensions. IndoorAtlas Floor Plans allows adding a floor mask - in which black are the areas allowing device positioning, while walls and obstacles, where the device position cannot be determined, are drawn in white.

\subsection{Position Calculation}

For positioning, IndoorAtlas uses pattern matching - in this, it uses measured data to overcome the cumulative position estimation error. The pattern matching technique consists of two phases:

- $\quad$ an offline training phase,

- $\quad$ an online tracking and positioning phase.

During the offline training phase, a database of variable magnetic density measurements at specific points in the building is built. The data collected by the magnetic sensor are sent to the IndoorAtlas positioning service via the API; here, they are stored in the fingerprint database from which the fingerprint map is then created for the mapped building. In IndoorAtlas terminology, this fingerprint map is the IndoorAtlas FloorPlans system. The IndoorAtlas FloorPlans web service evaluates and provides information on the following:

- convergence distance - the distance to travel to accurately position the device,

- the positioning error indicating the possible distance from the current location,

- map coverage indicating the area that has been mapped and can be used for positioning the device.

Based on information from [6], during this phase, it is important to secure in several conditions:

- The device calibration is required during fingerprinting on a regular basis to ensure data quality (the calibration screen will pop up automatically when calibration is necessary).

- Set start point and end point to draw a path on the floor plan.

- Stand at the start point, tap the record button, and then start walking immediately towards the end point.

- Stop recording when you reach the end point.

- Walk at a steady pace (without changing the speed too much) and in a straight line following the path.

- $\quad$ Keep the phone directed straight ahead in front of the person performing the operation.

- Keep the device at least $50 \mathrm{~cm}$ away from the wall. 
Figure 4 shows an example of the convergence distance in the mapped part of the building.

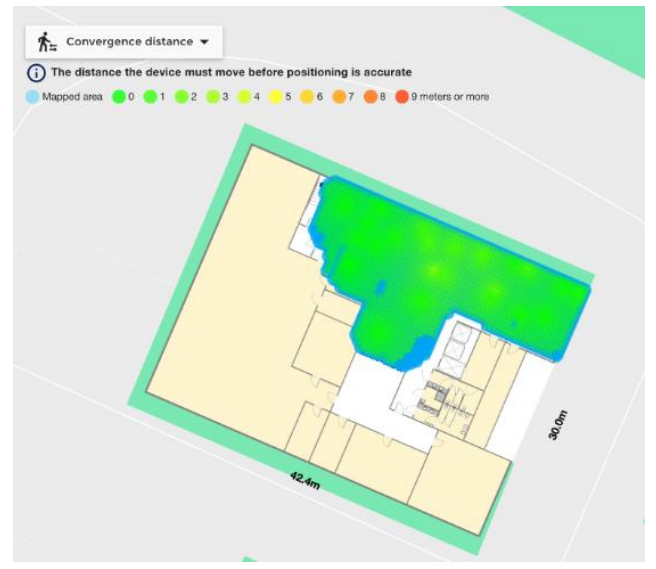

Figure 4

Convergence distance in the mapped part of the building

The data contained in the fingerprint database serve as reference points for the positioning phase. During the positioning phase, the current variable magnetic density, measured by the smartphone, is compared with all measurements stored in the fingerprint database. When the current data match the fingerprint in the database, it is highly probable that the user's position corresponds to the position where the fingerprint data were acquired from.

\subsection{Graph Nodes}

In the presented system, graph nodes will represent points on the respective floor of the building and the corresponding edges to create a logical map of the paths among the various points in the building. Each graph node (vertex) will have the following six parameters:

- an identifier, specifying a unique node,

- the node name,

- the node latitude,

- the node longitude,

- the floor of the node,

- a flag stating whether the node is a real position in the building or just a logical node needed for further calculations. 


\subsection{Graph Edges}

In the presented system, graph edges represent a path from a node to another node. Each edge has the following four parameters:

- $\quad$ an identifier, specifying a unique edge,

- the starting node of the edge,

- the final node of edge,

- the distance between the nodes, calculated as the actual distance between the geographical coordinates.

\subsection{Database of Geographical Graph Nodes}

The IndoorAtlas service also provides a web service called FloorPlans that allows the acquisition of geographic graph nodes. Also, it is able to create so-called transition points - the system determines the latitude and longitude of these. It is important to perform this step with utmost accuracy, as the transition points also reflect the real-world situation. This method is an implementation of the haversine formula:

$$
\begin{aligned}
& a=\sin ^{2}\left(\frac{\Delta \phi}{2}\right)+\cos \phi_{1} * \cos \phi_{2} * \sin ^{2}\left(\frac{\Delta \lambda}{2}\right) \\
& c=2 * \arctan 2(\sqrt{a}, \sqrt{(1-a})) \\
& d=R^{*} c
\end{aligned}
$$

where $\phi$ is the latitude, $\lambda$ is the longitude and $R$ is the ground radius in meters.

After calculating the distance between the current position and the compared node, the system verifies if the calculated distance is less than the smallest distance. If so, it is necessary to verify whether the edge connecting the node representing the current position and the compared node is valid. It may happen that the edge connecting the node closest to the current position and the node representing the current position pass through a wall. Such problems are solved programmatically by reading the downloaded IndoorAtlas FloorPlan plan as a bitmap. There, a path represents the edge connecting the compared node and the current position node is created. Later, the color of each pixel on the path is compared with the color used to highlight the walls. If the path does not contain wall color pixels, the edge is valid. If the previous condition is met, the shortest distance will be equal to the distance between the node of the current position and the currently compared node. 


\subsection{Results of the Propositioned Application Testing}

Positioning accuracy was tested by two phases of measurements that were carried out in the premises of the selected building in Košice, Slovakia. The first phase of the measurements was conducted within one room (short distance measuring) and the second one was measured within the building (long-distance measuring).

The first phase: two points were selected in a room and were localized using the IndoorAtlas service. These points were selected so that one point could be reached from another only by an imaginary line (depicted in blue). Data from the IndoorAtlas API helped to determine the positions of the points between the two selected points. Then, the data were extracted and used to create the graphs of the positions achieved. The measurements were carried out in two different rooms. The accuracy of the first measurement is shown in Figure 5, while the accuracy of the second measurement is depicted in Figure 6.

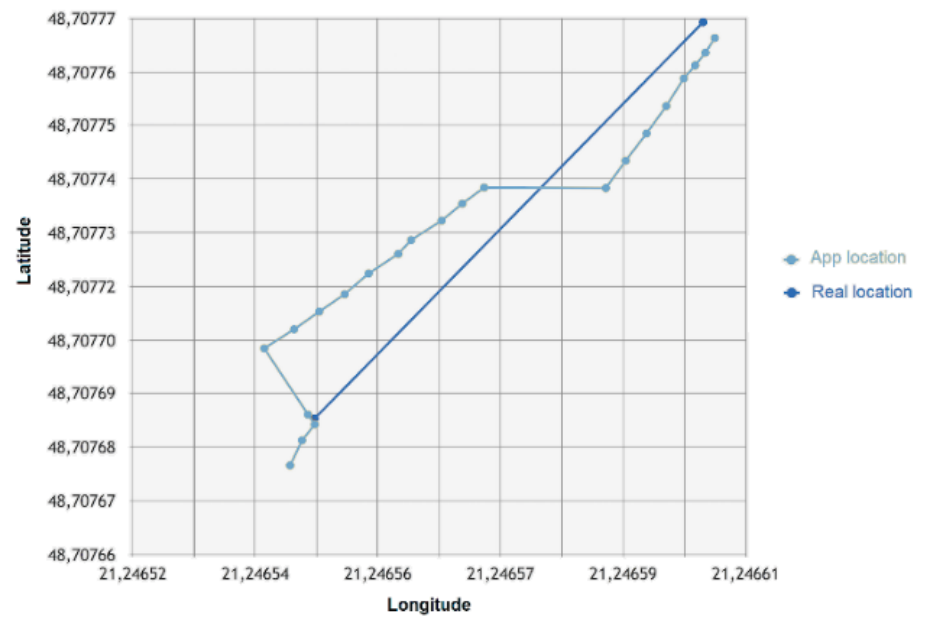

Figure 5

The first measurement of the phase one 


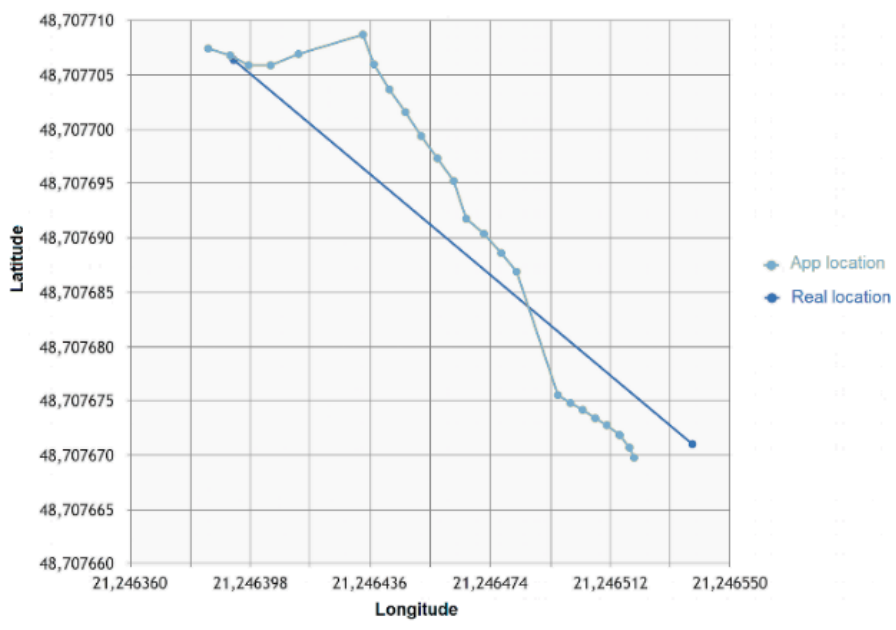

Figure 6

The second measurement of the phase one

Due to the small indoor distances, the curvature of the Earth need not be taken into account, so the measured distances may be mapped to the Cartesian coordinate system. Then, the distance of the individual points from the line representing the real trajectory of movement is calculated. The distance of the points from which it was not possible to construct a perpendicular to the particular segment of the actual trajectory is calculated as the distance of the particular point from the start point or end point of the direct trajectory. The distance of the points, from which it was possible to construct a perpendicular to a particular segment of the real trajectory is calculated using the distance of the particular point from the line, expressed as follows:

$d=\frac{\left|A \cdot M_{x}+B \cdot M_{y}+C\right|}{\sqrt{A^{2}+B^{2}}}$

The linear equation was constructed using the start and end points of the straight path. The distance of the points from the line was expressed in degrees and therefore had to be converted to centimeters using the haversine formula.

In the first measurement of phase one, the average deviation from the actual travel path was $80.72 \mathrm{~cm}$. In the second measurement of phase two, the deviation amounted to $58.24 \mathrm{~cm}$. The total mean deviation of the two measurements of phase one was $69.48 \mathrm{~cm}$. This deviation may be due to several factors:

- although during testing, the authors attempted to move between the two selected points as directly as possible with the smart device placed in an upright position, perpendicular to the chest, there is still a chance of inaccurate measurements due to imperfect straight forward movement, 
- measurement deviations may also be affected by electromagnetic waves present by nearby electrical equipment;

- measurements were not performed using a device that IndoorAtlas recommends for positioning.

The second phase: the theory behind the second phase of the measurements is pretty much the same as phase one except the fact that the conducted experiments were held within the whole building across the various rooms. Each of the measurements in this phase brings similar results to phase one and exact trajectories are shown in Figure 7 and Figure 8.

In the first measurement of the phase two, the average deviation from the actual travel path was $75.15 \mathrm{~cm}$. In the second measurement of the phase two, the deviation amounted to $81.63 \mathrm{~cm}$. The total mean deviation of the two measurements of phase two was $78.39 \mathrm{~cm}$. This deviation may be due to the same factors mentioned earlier. 


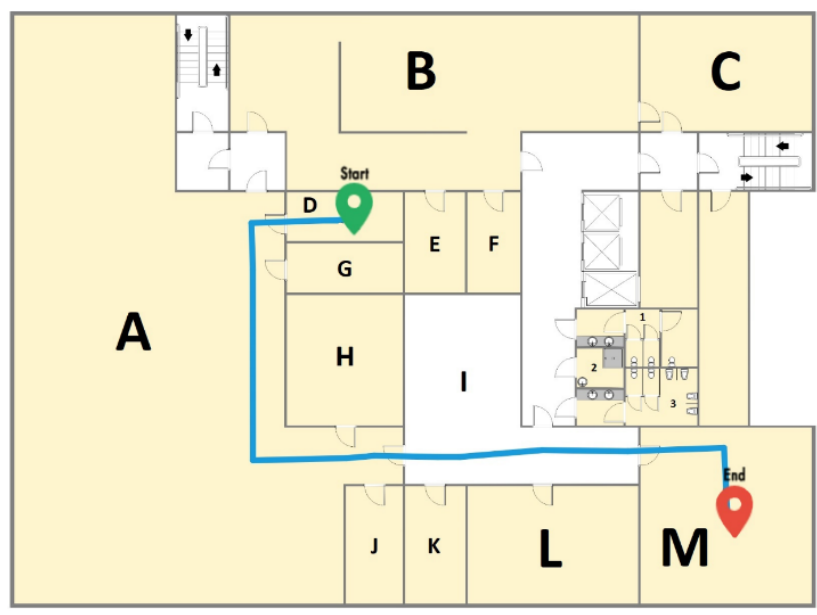

Real floor plan

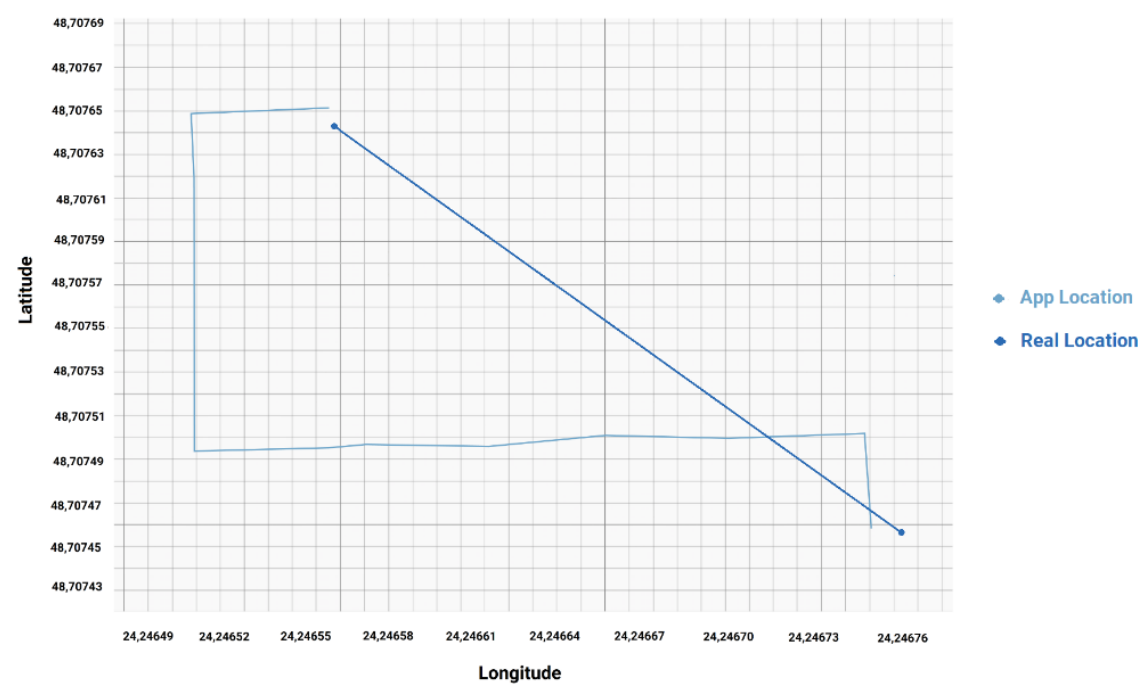

Figure 7

The first measurement of the phase two 

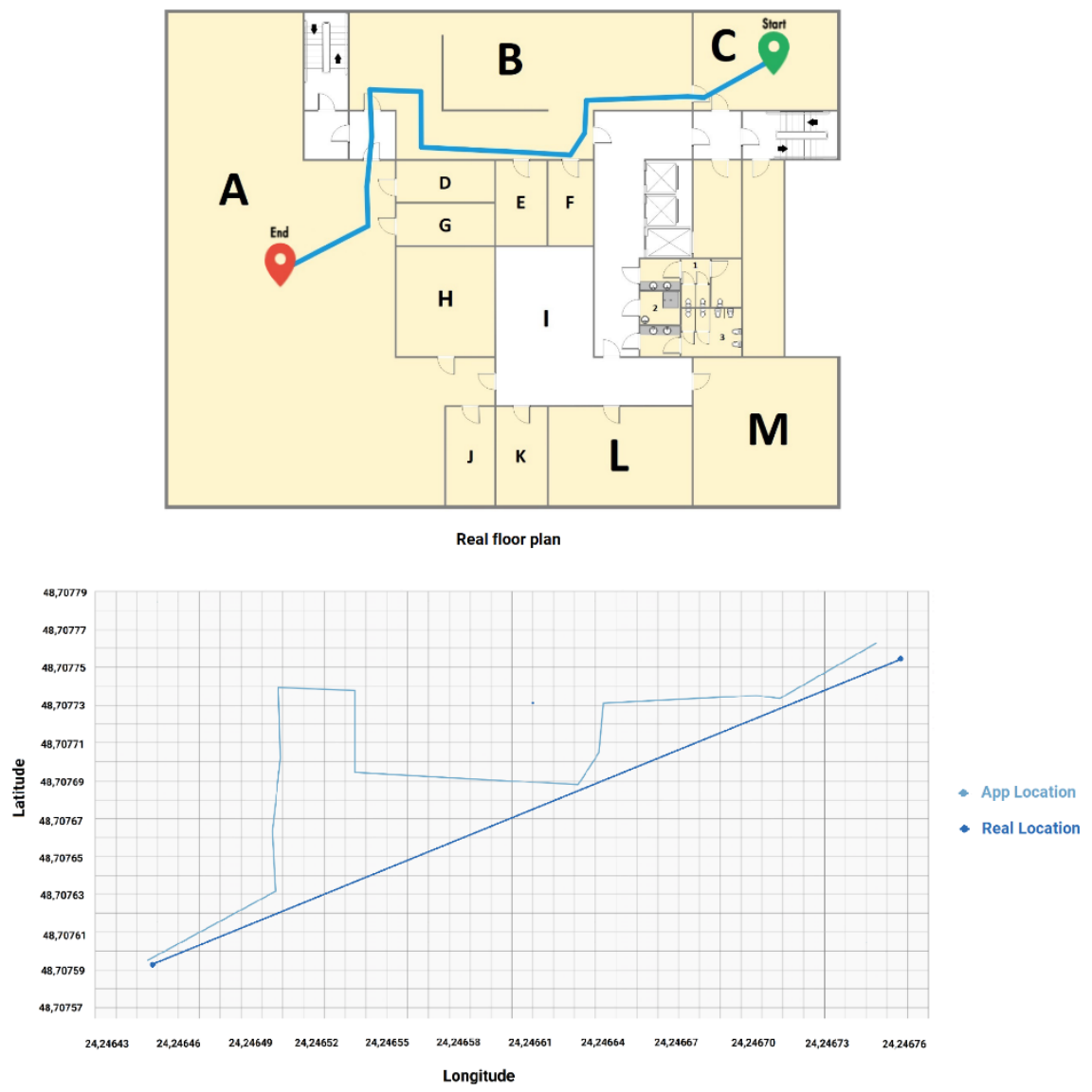

Figure 8

The second measurement of the phase two

\section{Achieved Results}

All the measurements were conducted within the selected building in Košice, Slovakia. During the process, there was a constant indoor ambient temperature of $23{ }^{\circ} \mathrm{C}$. As mentioned in Section 4, Table 1, the producer of the IndoorAtlas library gives the accuracy of the library between $0.1 \mathrm{~m}$ to $2 \mathrm{~m}$, which was successfully confirmed by our experiments. Table 2 below shows the complete results of the experimental part of the paper. 
Table 2

Complete results of the measurements

\begin{tabular}{|c|c|c|c|}
\hline Deviation & 1. measurement & 2. measurement & mean \\
\hline Phase one & $80.72 \mathrm{~cm}$ & $58.24 \mathrm{~cm}$ & $69.48 \mathrm{~cm}$ \\
\hline Phase two & $75.15 \mathrm{~cm}$ & $81.63 \mathrm{~cm}$ & $78.39 \mathrm{~cm}$ \\
\hline All & & & $73.935 \mathrm{~cm}$ \\
\hline
\end{tabular}

\section{Conclusions}

The presented article proposes an experimentally verified indoor navigation solution based on positioning using the Earth's magnetic field. Using the magnetic sensors present in mobile smart devices, the resulting program measures the properties of the surrounding magnetic field and then integrates it with the IndoorAtlas positioning service to determine the position of the device. The location is calculated by comparing the current data collected by the smart device sensor and the data stored in the IndoorAtlas database as Floor Plans. The shortest possible path is calculated using Dijkstra's algorithm. In the testing process of the proposed experimental solution, two phases of the measurements were performed with the results summarized in Section 8 . The producer of the IndoorAtlas library gives the accuracy of $0.1 \mathrm{~m}$ to $2 \mathrm{~m}$ which was successfully confirmed by the conducted experiments. In future testing and fine-tuning, the following steps would be beneficial to the application, to allow the most precise positioning:

- Since measurements were made only using a single smart device type, future research could include measurements using several devices equipped with magnetic sensors. Later, it individual measurements could be compared to determine which smart device and/or magnetic sensor is more useful in this type of navigation.

- It would be useful to compare the performance and calibration of the magnetic sensors of smart devices and to select devices having the sensors with the highest sensitivity and also suitable for interaction with the IndoorAtlas service.

- Verification or elimination of the assumptions mentioned in section 5.6 regarding the possible impacts on measurement accuracy would be beneficial.

- It would be worth trying to implement solution mechanisms in conjunction with other algorithms designed to find the shortest path in the graph.

- It would also be appropriate to implement security mechanisms [17] [18]. 


\section{Acknowledgement}

This work was supported by the Faculty of Electrical Engineering and Informatics at the Technical University of Košice under contract No. FEI-2021-76: A modern interpreter of predicate linear logic formulas.

\section{References}

[1] M. P. Wylie, J. Holtzman, "The non-line of sight problem in mobile location estimation," in Proc. of $5^{\text {th }}$ International Conference on Universal Personal Communications (ICUPC), MA, USA, 1996, pp. 827-831

[2] C. Hsieh, J. Chen and B. Nien, "Deep Learning-Based Indoor Localization Using Received Signal Strength and Channel State Information," in IEEE Access, Vol. 7, pp. 33256-33267, 2019

[3] N. Ádám, A. Baláž, E. Pietriková, E. Chovancová, and P. Fecil’ak, “The Impact of Data Representationson Hardware Based MLP Network Implementation," Acta Polytechnica Hungarica, Vol. 15, No. 2, pp. 69-88, 2018

[4] L. Vokorokos and N. Ádám, "Modeling of feedforward neural network in PAHRA architecture," in Proceedings of the $9^{\text {th }}$ WSEAS International Conference on Simulation, Modelling and Optimization, Budapest, Hungary, 2009, pp. 446-451

[5] H. Falaki, et al, "Diversity in smartphone usage," in Proc. of the $8^{\text {th }}$ International Conference on Mobile Systems, Applications, and Services, CA, USA, 2010, pp. 179-194

[6] IndoorAtlas, "Build seamless location-based experiences today," cited on $5^{\text {th }}$ of May 2019, Available online: <https://www.indooratlas.com/>

[7] B. V. Cherkassky, A. V. Goldberg, T. Radzik, "Shortest paths algorithms: Theory and experimental evaluation," in: Mathematical programming, Vol. 73, No. 2, 1996, pp. 129-174

[8] R. F. Brena, J. P. García-Vázquez, C. E. Galván-Tejada, D. MuñozRodriguez, C. Vargas-Rosales, and J. Fangmeyer, "Evolution of indoor positioning technologies: a survey," Journal of Sensors, Vol. 2017, Article ID 2630413, 21 pages, 2017

[9] H. Bay, T. Tuytelaars, and L. Van Gool, "SURF: Speeded Up Robust Features," in Computer Vision - ECCV 2006, Berlin, Heidelberg, 2006, pp. 404-417

[10] M. Afzalan and F. Jazizadeh, "Indoor Positioning Based on Visible Light Communication: A Performance-Based Survey of Real-World Prototypes," ACM Comput. Surv., Vol. 52, No. 2, May 2019, doi: 10.1145/3299769

[11] M. Kaluža, K. Beg, B. Vukelić: Analysis of an indoor positioning systems Zbornik Veleučilišta u Rijeci, Vol. 5 (2017), No. 1, pp. 13-32 
[12] S. Nishiguchi et al., "Reliability and validity of gait analysis by androidbased smartphone," Telemedicine and e-Health, Vol. 18, No. 4, pp. 292296, 2012

[11] D. K. Son, E. Cho, I. Moon, Z. Ghassemlooy, S. Kim, and C. G. Lee, "Simultaneous transmission of audio and video signals using visible light communications," EURASIP Journal on Wireless Communications and Networking, Vol. 2013, No. 1, p. 250, Oct. 2013, doi: 10.1186/1687-14992013-250

[12] Z. Yang, C. Wu, Z. Zhou, X. Zhang, X. Wang, and Y. Liu, "Mobility Increases Localizability: A Survey on Wireless Indoor Localization Using Inertial Sensors," ACM Comput. Surv., Vol. 47, No. 3, Apr. 2015, doi: $10.1145 / 2676430$

[13] H. Rashid and A. K. Turuk, "Dead reckoning localisation technique for mobile wireless sensor networks," in IET Wireless Sensor Systems, Vol. 5, No. 2, pp. 87-96, 42015

[14] J. Qi and G.-P. Liu, "A Robust High-Accuracy Ultrasound Indoor Positioning System Based on a Wireless Sensor Network," Sensors (Basel), Vol. 17, No. 11, p. 2554, Nov. 2017, doi: 10.3390/s17112554

[15] C. Gentner and T. Jost, "Indoor positioning using time difference of arrival between multipath components," International Conference on Indoor Positioning and Indoor Navigation, Montbeliard-Belfort, 2013, pp. 1-10

[16] S. Zhang, et al, "Magnetic declination and zonal wind effects on longitudinal differences of ionospheric electron density at midlatitudes," in Journal of Geophysical Research, Vol. 117, No. A8, USA, 2012, pp. A08329

[17] E. Chovancová et al., "Securing distributed computer systems using an advanced sophisticated hybrid honeypot technology," Computing and Informatics, Vol. 36, No. 1, pp. 113-139, 2017

[18] S. Kim, S. Ha, A. Saad and J. Kim, "Indoor positioning system techniques and security," 2015 Forth International Conference on e-Technologies and Networks for Development (ICeND), Lodz, 2015, pp. 1-4

[19] M. Kaluža and B. Vukelic, "Analysis of an indoor positioning systems," Zbornik Veleučilišta u Rijeci, Vol. 5, pp. 13-32, 2017, doi: 10.31784/zvr.5.1.2

[20] Boris V Cherkassky, Andrew V Goldberg a Tomasz Radzik. „Shortest paths algorithms: Theory and experimental evaluation”. In: Mathematical programming 73.2 (1996), s. 129-174

[21] A. Yassin et al., "Recent Advances in Indoor Localization: A Survey on Theoretical Approaches and Applications," in IEEE Communications 
Surveys \& Tutorials, Vol. 19, No. 2, pp. 1327-1346, Secondquarter 2017, doi: 10.1109/COMST.2016.2632427

[22] F. Zafari, A. Gkelias and K. K. Leung, "A Survey of Indoor Localization Systems and Technologies," in IEEE Communications Surveys \& Tutorials, Vol. 21, No. 3, pp. 2568-2599, thirdquarter 2019, doi: 10.1109/COMST.2019.2911558 